\title{
ULTRAVIOLET SPECTRAL IMAGING
}

\author{
James E. Neff \\ Joint Institute for Laboratory Astrophysics \\ Univ. of Colorado \\ Boulder, CO 80309-0440, USA
}

\begin{abstract}
I discuss the general problem of determining the spatial structure in the outer atmospheres of active, late-type stars. There are several major differences between the problems of imaging photospheres and of imaging chromospheres. Because of these differences, chromospheric spectral imaging must be based on a direct deconvolution of the observed emission line profiles. I present results based on IUE spectra of AR Lac (=HD 210334).
\end{abstract}

\section{INTRODUCTION}

High $\mathrm{S} / \mathrm{N}$ spectra are often required because one desires to measure a small signal against a bright background. One virtue of ultraviolet spectra of late-type stars is that the photospheric background is faint, allowing the measurement of small relative signals, even with moderate $\mathrm{S} / \mathrm{N}$ spectra. Stellar chromospheres and transition regions are best studied using ultraviolet emission line spectra.

Doppler imaging (see Vogt 1988) can be used to produce images of stellar photospheres using high-resolution, high $\mathrm{S} / \mathrm{N}$, optical absorption line profiles. In order to produce similar images of stellar chromospheres, similar procedures should be developed for the analysis of ultraviolet emission line spectra. In this talk, I discuss the general problem of producing images of rapidly rotating stars using observed spectral line profiles ("spectral imaging").

By combining spectral images of the photosphere and the chromosphere with other information about the structure of the corona (derived from radio and $\mathrm{x}$ ray observations), it is possible to develop a three-dimensional picture of stellar atmospheres. With such pictures, the size, location, and brightness of magnetic active regions can be studied as a function of time to determine stellar cycles and to measure differential rotation. It will then be possible to model the physical conditions within each atmospheric component separately. 


\section{ULTRAVIOLET VERSUS OPTICAL SPECTRAL IMAGING}

The goal of spectral imaging is to utilize all of the spatial information that exists in the observed line profiles from rapidly rotating stars. A discrete region of high contrast on the surface of a rapidly rotating star produces an effect on the line profile that moves from blue to red across the line profile as the star rotates. The amplitude of this velocity shift is $v_{\text {rot }} \sin i \cos \theta$ (where $v_{\text {rot }}$ is the rotational velocity of the star, $i$ is the inclination, and $\theta$ is the latitude of the region), if the region is on the stellar surface. When the region is on the central meridian, there is no velocity shift. By measuring the relative velocity of the feature with respect to the center of the line profile as a function of rotational phase, its longitude and latitude can be determined. If the region is spatially extended, the width of the spectral feature as a function of rotational phase can be used to determine the shape of the region on the stellar surface. For various reasons, a spectral image can not be derived by simply identifying the wavelength of "bumps" on the line profiles (e.g. Gondoin 1986).

While all spectral imaging techniques share these same fundamental principles, there are several noteworthy differences between optical (Doppler imaging) techniques (e.g., Hatzes 1988; Jankov 1987; Vogt 1987; Vogt and Penrod 1983; Vogt, Penrod, and Hatzes 1987) and ultraviolet techniques (Neff 1986; Neff 1987a, 1987b; Walter et al. 1987).

First and foremost, the physical properties of stellar chromospheres are not known. In fact, the goal of imaging is to determine these properties. The parameters needed to synthesize the intrinsic chromospheric profile are not available. For example, the variation in brightness of a chromospheric active region between the limb and disk center can not be determined until a region is spatially resolved. Available chromospheric models typically represent only a global average, not the physicaliy distinct regions that we expect to be present.

Second, in a given spectral line, most of the emission probably arises from discrete regions. This is emission above a very faint continuum. The more active the star, the brighter the discrete emission. By contrast, photospheric spots are dark regions on a bright background. Further, the photospheric lines become shallower the more rapidly the star rotates.

Because of these differences, chromospheric spectral imaging must be based on a direct deconvolution of emission line profiles. While tedious and difficult to automate, this procedure is capable of providing spatial information using spectra of lower $\mathrm{S} / \mathrm{N}$. 


\section{ULTRAVIOLET SPECTRAL IMAGING TECHNIQUE}

To illustrate the power and the limitations of this technique, I summarize the analysis of a series of spectra of AR Lacertae (=HD 210334). AR Lac is an eclipsing RS CVn binary system (K0 IV + G2 IV). The two stars rotate synchronously with the orbital period (1.98 days), yielding an equatorial velocity of $72 \mathrm{~km} \mathrm{~s}^{-1}$ for the $\mathrm{K}$ star and $39 \mathrm{~km} \mathrm{~s}^{-1}$ for the $\mathrm{G}$ star. Because the system is bright $\left(\mathrm{m}_{\mathrm{v}}=6.1\right)$ and because both stars are active, the system is an ideal candidate for ultraviolet spectral imaging.

We observed AR Lac over $80 \%$ of an orbital cycle in Sep. 1985 (Neff, Walter, and Rodonò 1986). Eighteen high-resolution $(\mathrm{R} \sim 20,000)$ spectra of the $\mathrm{Mg}$ II $\mathrm{k}$ $(2795 \AA)$ line and 18 low-resolution (6 $\AA)$ spectra covering the range 1150 to 1950 $\AA$ were obtained.

The 18 observed $\mathrm{Mg}$ II $\mathrm{k}$ line profiles can, at first glance, be characterized by two emission components at radial velocities roughly corresponding to the predicted orbital velocities of the two stars plus an unresolved interstellar absorption line. In the initial round of the analysis, three gaussian components are fitted to the observed profiles. The width of the interstellar component is constrained to be the instrumental width.

In order for these three-component fits to provide an appropriate model of the line profiles, (1) the velocities of the two emission components must match the predicted stellar velocities, (2) the widths of these components must be consistent with the stellar rotational velocities, and (3) the equivalent width of the interstellar absorption component must remain roughly constant. None of these conditions are met by the three-component fits.

Emission arising from a uniform distribution on a stellar surface will produce a symmetric profile centered at the stellar velocity. Large-scale non-uniformities will produce emission components that vary in wavelength due to the Doppler shift produced by the star's rotation. The net profile will be asymmetric, and a singlecomponent fit to this profile will not lie at the stellar velocity. Therefore by fitting the observed profiles with a symmetric component centered at the stellar velocity plus additional components to account for the residual emission, the non-uniform distribution can be mapped. Neff (1987a) and Walter et al. (1987) describe this procedure in detail. In practice, the minimum number of additional components required to match the observed profiles are determined iteratively, using an interactive multiple-component fitting routine. The primary constraints in this procedure are the positions of the uniform stellar components and the interstellar absorption equivalent width, which should remain constant. Secondary constraints are the rough constancy of the stellar emission line widths and smooth point-to-point variation of the stellar flux. 


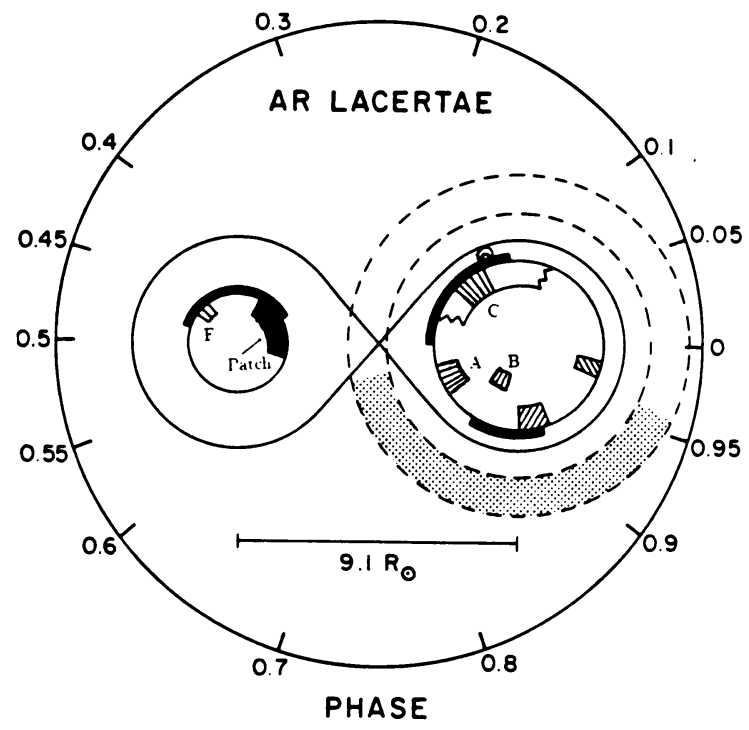

Figure 1: The latitudes, longitudes, and sizes of three plages on the $\mathrm{K}$ star (right), a flare on the G star (left), and the inactive region on the $G$ star are depicted schematically to the scale of the binary system. The unlabelled plages are regions identified in a series of spectra obtained in Oct. 1983 (Walter et al. 1987). The compact and extended coronal structure was determined by the $\mathrm{x}$-ray light curve obtained during eclipses in June 1980 by Walter, Gibson, and Basri (1983).

\section{THE SPATIALLY RESOLVED CHROMOSPHERE OF AR LACERTAE}

The resulting system model for AR Lac in Sep. 1985 (Figure 1) reveals five discrete chromospheric regions: (1) Plage A is located at longitude $205^{\circ}$, latitude $0^{\circ}$ on the $\mathrm{K}$ star (longitude $0^{\circ}$ corresponds to orbital phase 0.0 , when the $\mathrm{G}$ star is totally eclipsed). It likely extends well above the stellar photosphere. The region covers $6 \%$ of the stellar surface. (2) Plage B is located at longitude $240^{\circ}$, latitude $\pm 50^{\circ}$ on the $\mathrm{K}$ star. It covers $2 \%$ of the stellar surface. (3) Plage $\mathrm{C}$ is located at longitude $130^{\circ}$, latitude $0^{\circ}$ on the $\mathrm{K}$ star. It likely extends well above the photophsere. It covers $9 \%$ of the stellar surface. (4) The $G$ star is chromospherically inactive between longitudes $335^{\circ}$ and $75^{\circ}$. (5) A flare was observed on the G star. The flaring region covered at most $2 \%$ of the stellar surface. In addition, the global (uniform) flux from the $\mathrm{K}$ star is $40 \%$ brighter from the trailing hemisphere than from the leading hemisphere. This information is presented as a series of images by Neff $(1987 a, 1987 b)$. 


\section{CONCLUSIONS}

Very detailed images of stellar photospheres can be derived by synthesizing a line profile from an assumed brightness distribution and then varying this distribution until the synthetic profile matches the observed profile. Unfortunately, a similar procedure can not be used to image stellar chromospheres. Nevertheless, a non-uniform chromospheric distribution manifests itself in the observed profiles. Because these non-uniformities are large scale and because the photospheric background is fainter in the ultraviolet, chromospheres can be imaged by a careful deconvolution of the line profiles. Higher quality data obtained with the Goddard High Resolution Spectrograph on the Hubble Space Telescope will allow more detailed images to be derived. It should also be possible to apply the same techniques to ground-based spectra of the Ca II K lines. Because of poor instrumental throughput and detector sensitivity, this is currently a difficult task. The $\mathrm{H} \alpha$ emission line is poorly suited for spectral imaging, primarily because it is substantially broadened in these stars by non-rotational mechanisms.

\section{REFERENCES}

Gondoin, P. 1986, Astr. Ap., 160, 73.

Hatzes, A.P. 1988, these proceedings.

Jankov, S. 1987, unpublished thesis, Universite Paris-Meudon.

Neff, J.E. 1986, in Proc. SHIRSOG Science Working Group, NSO.

Neff, J.E. 1987a, Ph.D. thesis, University of Colorado, Boulder.

Neff, J.E. 1987b, in Proc. Fifth Cambridge Workshop on Cool Stars, Stellar Systems, and the Sun, eds. J.L. Linsky and R.E. Stencel, Springer-Verlag, in press.

Neff, J.E., Walter, F.M., and Rodonò, M. 1986, in New Insights in Astrophysics: Eight Years of Astronomy With IUE, ESA SP-263, p. 153.

Vogt, S.S. 1988, these proceedings.

Vogt, S.S., and Penrod, G.D. 1983, Pub. Astr. Soc. Pacific, 95, 565.

Vogt, S.S., Penrod, G.D., and Hatzes, A.P. 1987, Ap. J., submitted.

Walter, F.M., Gibson, D.M., and Basri, G.S. 1983, Ap. J., 267, 665.

Walter, F.M., Neff, J.E., Gibson, D.M., Linsky, J.L., Rodonò, M., Gary, D.E., and Butler, C.J. 1987, Astr. Ap., in press. 


\section{DISCUSSION}

JUDGE A general comment about chromospheric modelling. There has been much discussion about the inhomogeneous structure of stellar chromospheres for which there is much evidence in the sun and in some parts of the HR diagram, Jim has presented a graphic demonstration of the inhomogeneous chromosphere of the AR LAC K-star component. This star is an active star. To take the devil's advocate I suggest that, for 'normal' late-type stars one can still usefully apply one-component modelling techniques, provided one is careful not to over-intexpret the data in particular, for the least active stars (single giants later than $\approx$ kO) there is some indication that acoustic, and not magnetic heating could be responsible for the abserved chromospheric emission ( $\mathrm{I} \cdot \mathrm{m}$ thinking of the 'basal fluxes' identified by the Utrecht group) which probably could not lead to the kind of discrete structures discussed here. The assumptions of one-component plane parallel models are clearly oversimplifications which nevertheless remain to be properly tested by abservations in most cases, therefore these modelling techniques should still be pursued as useful first attempts at understanding spectra which, taking the case of cool, 'non-solar' stars, as an example, are only beginning to be understood.

LINSKY The Sun is a very inactive star yet many components are needed to explain the range of spectroscopic features observed in integrated light. While it may be true that the cool single giants have simple, nonmagnetically heated atmospheres, this is not proven in any way. I agree that it makes sense at this time to model the atmospheres of these very quiet stars with one atmosphere component, however we should keep in mind that this way be a gross oversimplification due to the possible presence of radial and nonradial oscillations, acoustic waves, and large scale convective cells even if magnetic structures do not exist.

FoING What is the accuracy in velocity shift and width determination and how does it translate in terms of accuracy in longitude, latitude and size of plage areas?

NEFF I have derived the longitudinal extents of the plages by assuming that the deconvolved line width at the narrowest plage profile is due solely to rotational smearing. In fact, there is probably a substantial intrinsic width of the plage profile, so my size determination is an upper limit. The assumption of circular plages introduces further uncertainty. Therefore, the formal uncertainties in the analysis are much smaller than the real uncertainties. The longitudes are well determined, say \pm 100 , by the phase of their central meridian passage. Their latitudes are determined by the amplitude of their radial velocity curves. Unfortunately, the height of the plage and differential rotation also affect the amplitude.

MARCY What fraction of the stellar surfaces are covered by active regions in the AR Lac system?

NEFF If you assume that the $K$ stax is uniformly covered by plages smaller than but otherwise similar to the three that are resolved, then the total filling factor is about 25\%. The global $G$ star emission cannot be due to a distribution of similar plages, because the line 
ratios (particularly C IV/C II) are different.

LINSKY I would like to point out that the Doppler imaging technique provides information on the longitude, latitude, and area of active regions on stars. The last quantity is extremely important because it permits us to infer the surface brightness of emission lines in active regions and thus to infer chromospheric models of the active regions.

NEFP None.

PITZPATRICK How is the strength of the interstellar absorption component constrained in the fitting of the multiple line components? NEFF The strength of the interstellar absorption is not known a priori. However, the equivalent width should remain constant. This provides an important constraint on the fitting procedure. If the interstellar equivalent width in a given fit is much smaller than the mean determined by all other fits, then the emission level at that wavelength from the system must be underestimated, so the fit is rejected. 